\title{
Espasmo Hemifacial: relato e comparação entre dois casos.
}

\author{
Hemifacial spasm: report and comparison between two cases
}

Peruzini, G. A.; Wienen, C. M.; Barros, F. C. $\$$ Monteiro, G. N. ; da Silva, L. S. $\$$ Pinto, M. R.de S. ; de Macedo,R. P.; Samel, V. G.; Tiel, C

Como citar esse artigo. Peruzini,G.A.;

Wienen, CM; Barros, FC; Monteiro, GN; da Silva, LS; Pinto, MRS de Macedo, RP; Samel, VG; Tiel, C. Espasmo Hemifacial: relato e comparação entre dois casos. Revista de Saúde. 2018 Jul./Dez.; 09 (2): 21-26.

\begin{abstract}
Resumo
O Espasmo Hemifacial (EH)é caracterizado por contrações tônico-clônicas musculares involuntárias dos músculos inervados pelo sétimo par craniano. O presente estudo tem o intuito de relatar dois casos de $\mathrm{EH}$ de etiologias distintas, descrevendo os principais sinais, sintomas e o tratamento da patologia.No caso 1, foi constatada a presença de uma alça vascular (EH secundário),através da Ressonância Nucelar Magnética (RNM). Após insucesso de terapêutica anterior, houve introdução da toxina botulínica, obtendo sucesso no tratamento. Já no caso 2, a paciente apresentou Tomografia Computadorizada (TC) de crânio e RNM sem alterações (EH idiopático), também apresentando melhora ao tratamento com a neurotoxina do tipo A.O tratamento pôde incluir resultados curativos por intermédio de cirurgia microvascular descompressiva e sintomáticos. Sendo assim, é imprescindível a eficácia diagnóstica e terapêutica, o que contribui significativamente à qualidade de vida dos pacientes, que sofrem de baixa autoestima, depressão e interação social prejudicada.
\end{abstract}

Palavras-chave: Espasmo hemifacial; neurologia; nervo facial; diagnósticos diferenciais

\begin{abstract}
Hemifacial spasm (HS) is characterized by involuntary muscle tone-clonic contractions of the muscles innervated by the seventh cranial nerve. The present study intends to report two cases of HS of different etiologies, describing the main signs, symptoms and treatment of the pathology. In case 1, the presence of a vascular loop (secondary HS) was observed throughmagnetic resonance imaging (MRI). After the failure of the previous therapeutics, the botulinum toxin was introduced, achieving success in the treatment. In case 2, a patient presented cranialcomputerized tomography (CT) and MRI without changes (idiopathic HS), also showing improvement to the treatment with neurotoxin type A. Treatment presented curative effects through decompressive and symptomatic microvascular surgery. This way, diagnostic and therapeutic efficacy is essential, which contributes significantly to patients' quality of life, who suffer from low selfesteem, depression and impaired social interaction.
\end{abstract}

Keywords: Hemifacial spasm; neurology; facial nerve; differential diagnoses;

\section{Introdução}

O espasmo hemifacial (EH) é um distúrbio de movimento caracterizado por contrações tônico-clônicas musculares involuntárias repetitivas dos músculos inervados pelo sétimo par craniano e que independem da vontade do paciente.Podem ser desencadeadas pelos movimentos faciais e exacerbadas sobre tensão psíquica, não podendo ser controladas voluntariamente ${ }^{1}$. $\mathrm{O}$ sítio mais comum de contraturas é a região do músculo orbicular dos olhos ${ }^{2}$. O EH pode ser classificado conforme a patologia de base em: idiopático (ou primário) e secundário. $\mathrm{O} \mathrm{EH}$ primário decorre de uma compressão benigna do nervo facial dentro ou próximo a sua zona de entrada ou saída do tronco cerebral ${ }^{13}$.
$\mathrm{O}$ EH secundário decorre daparalisia facial (EH pósparalítico), lesão do sétimo nervo, desmielinização e insultos vasculares cerebrais ${ }^{4}$.

É uma condição crônica, que raramente tem remissão espontânea, cuja incidência é de aproximadamente dois novos casos por milhão de habitantes por ano, ocasionando uma prevalência de 29,5 casos por 100.000 habitantes $^{1,4,5}$. Apesar da maioria dos enfermos com EH não apresentar redução de expectativa de vida, frequentemente sofrem de baixa autoestima, depressão e interação social prejudicada, sendo por isso o EH um fator que influencia negativamente o bemestar do paciente ${ }^{4,5}$.São comumente exacerbados pela fadiga e pelo estresse, além de permanecerem durante o sono, sendo um dos poucos distúrbios extrapiramidais a possuir essa característica marcante ${ }^{6}$.

\footnotetext{
Afiliação dos autores:

\$ Discente da Universidade de Vassouras; Vassouras/RJ;

$\dagger$ Docente da Universidade de Vassouras; Vassouras/RJ

* Email de correpondência: giovannalvesp@gmail.com;
} 
O EH acomete, geralmente, indivíduos de 43 a 53 anos e a sua prevalência é maior no sexo feminino do que no masculino, sendo o lado esquerdo mais comumente acometido que o direito ${ }^{4,8}$. Pode dificultar a visão binocular, e, em situações extremas, pode culminar com amaurose funcional temporária ${ }^{8}$. A sua evolução é céfalo-caudal, iniciando-se unilateralmente e, posteriormente, em toda a hemiface ${ }^{9}$. A sua patogênese ainda é de causa obscura e controversa e sua etiologia inclui desde a constrição do nervo facial pelo arranjo vasculara tumores cerebrais ${ }^{4}$.

$\mathrm{O}$ EH deve ser distinguido de outras patologias que causam movimentos involuntários faciais, como a mioquimia facial, contratura facial, parética e espástica, os tiques, a Síndrome de Meige, o Blefarospasmo, o Espasmo Facial Psicogênico e a Discinesia Tardia ${ }^{4,5}$. Haja visto que o EH primário decorre de um conflito entre o nervo facial e um vaso, deve ser distinguido destas causas mimetizadoras e, ainda, das causas secundárias, incluindo o EH pós-paralisia facia ${ }^{10}$.

Os recursos terapêuticosdo $\mathrm{EH}$ constamde várias opções, como medicamentos (benzodiazepínicos, imipramina, fenitoína), procedimentos cirúrgicos extracranianos (injeções de álcool ou de cloreto de procaína no nervo facial e rizotomias), técnicas de anastomose do ramo distal do nervo facial, cirurgia intracraniana (descompressão microvascular do sétimo par dos nervos cranianos) e a neurotoxina botulínica do tipoA que é a primeira escolha terapêutica ${ }^{4}$ e causa uma denervação química pela restrição da liberação de acetilcolina na junção neuromuscular ${ }^{6}$. Os pontos de aplicaçãosão, geralmente, na região do músculo orbicular dos olhos e também na região zigomática ${ }^{11}$. A recuperação dos sintomas varia de $82 \%$ a $100 \%{ }^{2}$. Os efeitos colaterais mais comuns são fraqueza palpebral, ptose e o enfraquecimento dos músculos peribucais. Lacrimejamento, secura ocular e diplopia são menos comuns ${ }^{4}$. $\mathrm{O}$ efeito benéfico dura cerca de três a quatro meses ${ }^{4}$. O tratamento definitivo para o EH é a técnica conservadora de Cirurgia Microvascular Descompressiva (CMD) cujo potencial de cura é de $85 \%$ a $95 \%$ dos pacientes ${ }^{12,13,14}$. No entanto, nem todos os pacientes submetidos a CMDirão receber alívioimediato, sendo comum que ocorra melhora das contrações algum tempo após a realização da cirurgia ${ }^{13,15}$.

O presente artigo objetivou relatar dois casos de EH com diferentes etiologias cuja finalidade foi analisar os tratamentos utilizados para regressão e cura da doença, bem comoabordar as principais patologias que devem ser diferenciadas do EH. A escolha do tema deve-se ao fato da doença ser negligenciada em nível semiológico, ao estigma e aos impactos sociais sobre a vida do paciente, além das diferentes etiologias e manifestações encontradas nos dois pacientes apresentados.

\section{Material e Métodos}

Este relato de caso se baseou na coleta de dados mediante a entrevista com os pacientes, registros fotográficos dos métodos diagnósticos e revisão dos prontuários, seguido de breve revisão literária com base em artigos encontrados nas seguintes bases: PubMed, Google Acadêmico, Scielo e Lillacs.

\section{Descrição dos casos}

Caso 1: JMA, 46 anos, masculino, pardo, natural de Minas Gerais, hemofílico. Paciente do Hospital Municipal de Porto Real relatou que há quatro anos iniciou contrações involuntárias na pálpebra esquerda e, posteriormente, na boca ipsilateralmente. As contrações tornavam-se mais evidentes ao conversar, ler e em momentos de inquietação, e reduziam-se quando estava relaxado. Negou dores e alterações da visão, mas referiu grandeincômodo ao conversar com outras pessoas, devido ao quadro de contrações. Procurou o médico da Unidade Estratégia Saúde da Família (UESF),sendo encaminhado para um oftalmologista. Seus exames oftalmológicos estavam normais, sendo, posteriormente, encaminhado para um neurologista. Por este, foram solicitados diversos exames, incluindo uma Ressonância Nuclear Magnética (RNM), que sugeriu a existência de uma alça vascular (Figura 1).Fez uso deCarbamezepina $200 \mathrm{mg} 1$ comprimido (cp) 8/8h e Clonazepam 2 mg 1 $\mathrm{cp} /$ diavia oral durante um mês, não havendo melhora do quadro. Um mês após a falha terapêutica com os medicamentos iniciou o tratamento com a neurotoxina botulínica do tipo Avia intramuscular - 20 unidades internacionais (UI) no músculo orbicular dos olhos em quatro pontos, 5 UI no músculo zigomático maior, 5 UI no músculo risório e 5 UI no músculo mentoniano. Até o dia 27/10/2016, havia feito cinco aplicações e com o tratamento com a toxina houve melhora do quadro. $\mathrm{O}$ exame neurológico indicou contrações clônicas dos músculos da hemiface esquerda, possivelmente os músculos: orbicular do olho, zigomático e mentoniano; os demais aspectos do exame foram normais (Figuras 2 e 3). O paciente continuou em acompanhamento apenas pelo neurologista.

Caso 2: MASC, 68 anos, sexo feminino, parda, casada, destra, natural de Minas Gerais e residente em Barra Mansa há 50 anos.Paciente do Ambulatório do Hospital Barra Mansa, relatou que há 16 anos teve um evento de afasia por 5 minutos concomitante com uma sensação de mal-estar e um quadro de hipertensão arterial. Procurou atendimento especializado e iniciou tratamento com extrato de Ginkgo Biloba $80 \mathrm{mg} 1 \mathrm{cp} / \mathrm{dia}$, Clonazepam $2 \mathrm{mg} 1 \mathrm{cp} /$ dia e Flunarizina $10 \mathrm{mg} 1 \mathrm{cp}$ de 12/12h. 


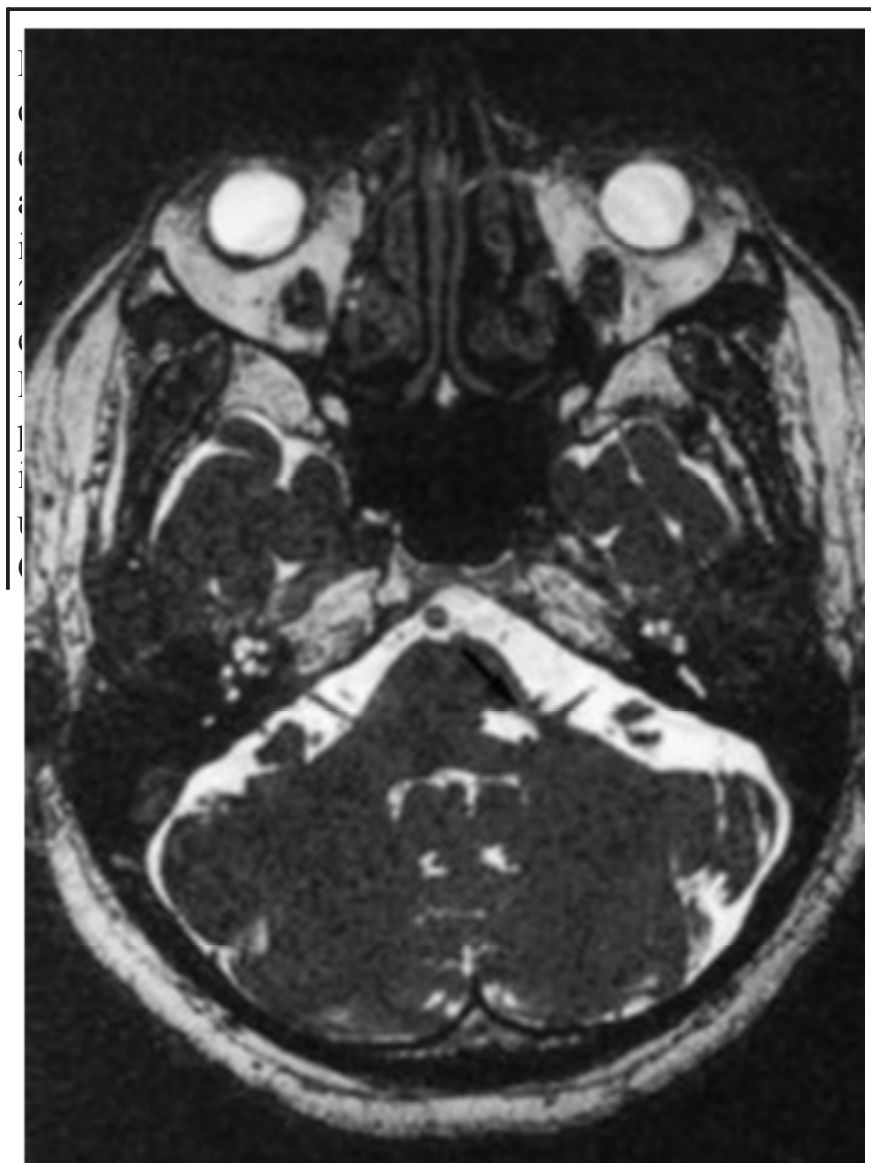

Figura 1. RNM de crânio em T2: alça vascular à esquerda

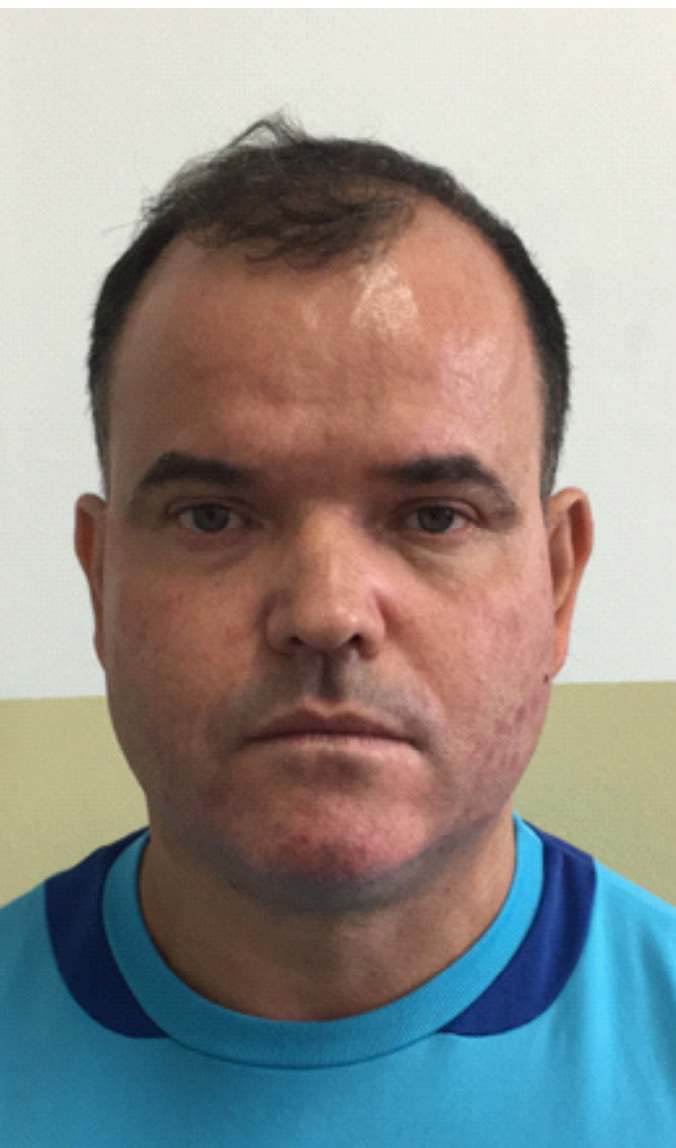

Figura 2. Paciente em repouso
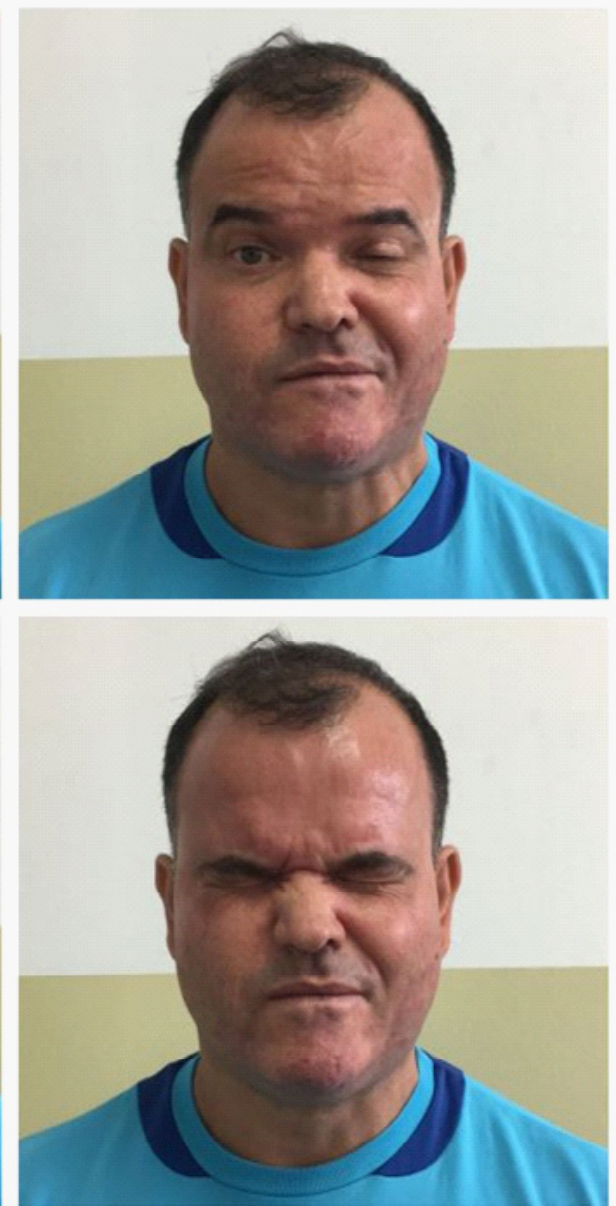

Figura 3. Exame do VII nervo com exacerbação das distonias faciais com a ativação muscular dos músculos inervados pelo nervo facial 
resultadosnão apresentaram nenhuma alteração em ambos. Iniciou entãotratamento sintomático, com aplicação de neurotoxina botulínica tipo A, sendo a primeira aplicação em 2013, observando uma melhora significativadas crises. $\mathrm{O}$ exame neurológico mostrou contrações tônico-clônicas involuntárias dos músculos da hemiface direita(Figuras 4 e 5). Os demais exames neurológicos realizados apresentaram-se normais.

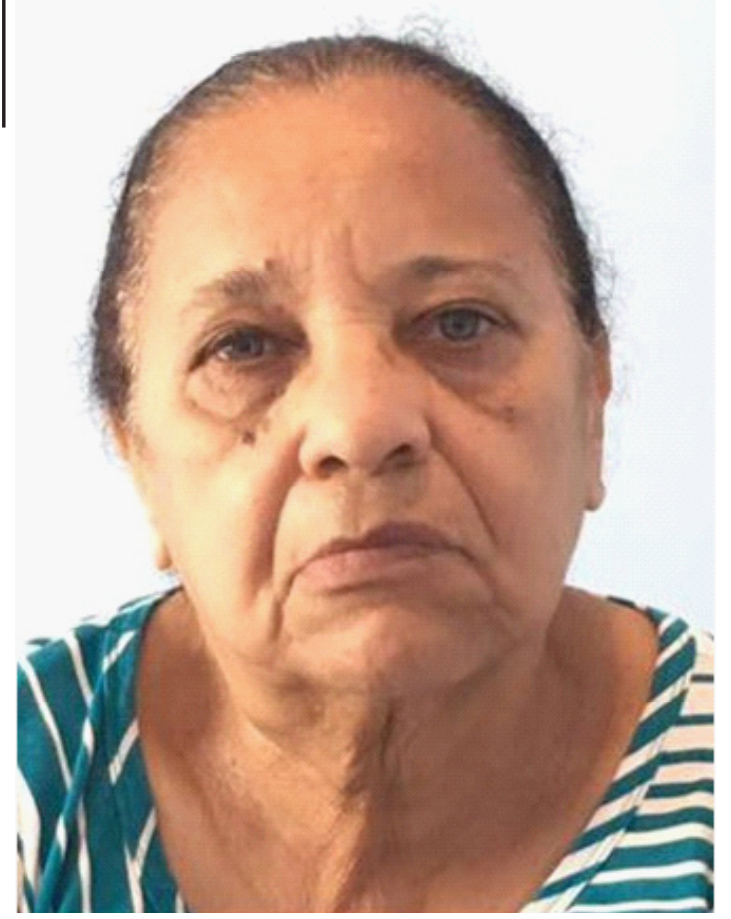

Figura 4 - Paciente em repouso

\section{Discussão}

O EH é um distúrbio do movimento, indolor, com condição crônica, caracterizado por contrações tônico-clônicas involuntárias dos músculos faciais e pescoço, fato que traz desconforto para o indivíduo acometido e queda da autoestima, além de uma série de limitações mediante tal acometimento? ${ }^{7}$.

É frequentemente causado pela constrição do VII nervo em sua zona de saída da raiz do tronco encefálico por alças vasculares ou aneurismas ${ }^{8}$. A predominância de acometimento se dá apenas em adultos, sendo mais frequente emmulheresetrazconsigo como característicaa incapacidade de supressão desse movimento, que reflete de maneira clara como a contração exacerbada do grupamento muscular independente da vontade do indivíduo ${ }^{8}$.

Em grande parte dos casos, a contração dos músculos se dá de maneira sincronizada, espontânea da mesma maneira e possui a característica marcante dapersistência do distúrbio durante o sono ${ }^{4}$,. Podem ocorrer espasmos longos ao sefecharem os olhos, esticar a testa ou retrair o ângulo da boca9 .

$\mathrm{O}$ diagnóstico correto e a diferenciação do $\mathrm{EH}$ de

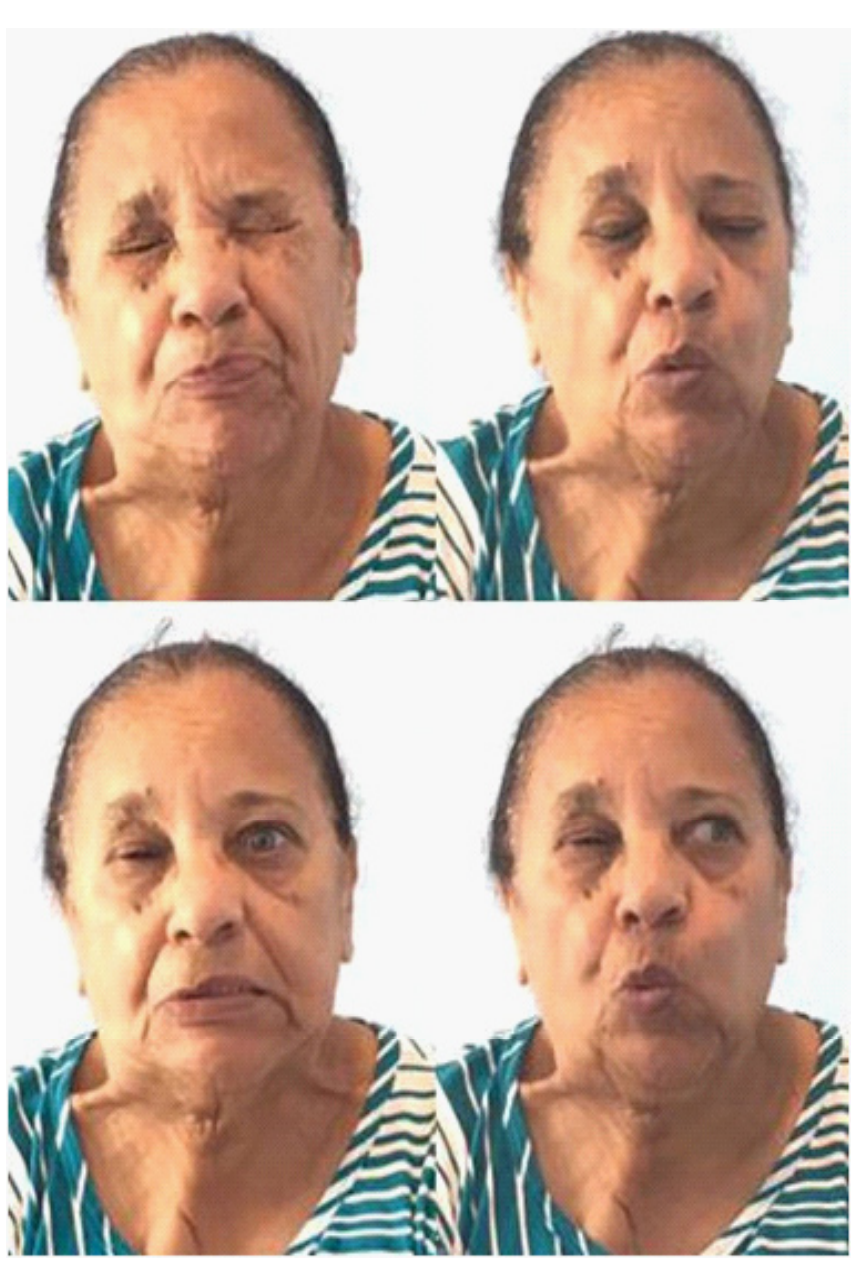

Figura 5 - Exame do VII nervo com exacerbação das distonias faciais com a ativação muscular dos músculos inervados pelo nervo facial

outros tipos de distonias são de extrema relevância para a terapêutica eficiente. Os principais tipos de distúrbios do movimento e suas características diferenciais são descritas na tabela ${ }^{15}$.

Conforme a patologia de base, o caso de EH no caso 1 resulta de um EH causado por uma alça vascular sobre o sétimo par craniano, ao nível da saída de sua raiz no tronco encefálico, levando a compressão do mesmo. O caso 2 descrito deste relato diz respeito ao EHidiopático e não há alteração perceptível nos exames de imagem. Vale destacar que o caso 1 é de um paciente do sexo masculino, fato menos prevalente ${ }^{3}$, entretanto este apresenta o lado esquerdo acometido, correspondendo a maior estatística, iniciando nos olhos e posteriormente no resto da hemiface. Já o caso 2 , é uma paciente do sexo femininoque apresenta o lado direito acometido, menos frequente, com início da alteração nos músculos zigomático maior, menor e risório e, posteriormente nos olhos.

O tratamento também difere nos dois casos relatados, sendo o primeiro, por ter uma causa definida e conhecida, a intervenção cirúrgica é a opção de tratamento. Entretanto, em vista do referido paciente ser portador de hemofilia, um distúrbio que implica diretamente na deficiência da coagulação sanguínea 
por carência de fatores de coagulação, o procedimento cirúrgico foi preterido. Essa cirurgia corresponde a uma descompressão da alça vascular na fossa média, sendo a operação feita sob anestesia geral por meio de uma incisão retromastoídea e craniotomia com uma janela de $3 \times 3,5 \mathrm{~cm}$ para que a compressão vascular possa ser identificada, a artéria ou artérias causadoras são mobilizadas e postas longe do nervo ${ }^{8}$.O problema mais frequente para tal procedimento é a disfunção permanente ou temporária do VII ou do VIII par craniano ${ }^{8,9}$. Como o caso 2 não apresentou uma causa estabelecida, só havia possibilidade de um tratamento paliativo, como por exemplo, a aplicação de toxina botulínica do tipo A com a finalidade de reduzir os sintomas das contrações involuntárias, para que a paciente tivesse ummelhor bem-estar e convívio social. O tratamento paliativo com a neurotoxina também foi amplamente utilizado e redime o espasmo por um período de 1 a 4 meses.

A toxina botulínica do tipo A age por bloqueio seletivo e reversível da junção neuromuscular, causando relaxamento da musculatura envolvida. A toxina inibe a liberação de acetilcolina das vesículas pré-sinápticas da placa neural, provocando uma neurectomia transitória e causando paralisia muscular ${ }^{6}$. É extremamente eficaz em aproximadamente 85 a $90 \%$ dos $\operatorname{casos}^{6,9}$. O tratamento medicamentoso com os medicamentos já citados também é utilizado e muitas vezes levam até a remissão total do $\mathrm{EH}^{8}$, entretanto em certos casos não há efeito algum, como ocorreu no paciente do primeiro caso.

O tratamento é dependente da etiologia da doençae consiste no procedimento cirúrgico de descompressão do nervo facial - para resolução do EH secundário- ouna administração de fármacos benzodiazepínicos com o intuito de amenizar as contrações exacerbadas. Há aindaa possibilidade da aplicaçãode toxina botulínica do tipo A com intervalo de 1 a 4 meses, visando oferecer um melhor bem-estar e conforto ao paciente com dada patologia. Vale destacar, que o tema ainda é pouco estudado e relatado, sendo importante a realização de novos estudos e pesquisas, a fim de descrever a patogênese da doença e opções terapêuticas, principalmente, para que o EH possa ser mais conhecido, melhor abordado e identificado.

\section{Conclusão}

Os casos relatados no presente artigo demonstram duas diferentes etiologias do EH: uma por compressão do VII par craniano por um arranjo vascular e outra de origem idiopática. Os fatores agravantes das contrações decorrem de desequilíbrios e tensões físicas e psíquicas como a ansiedade, fadiga e estresse, com persistência do distúrbio durante o sono. $\mathrm{O}$ diagnóstico é feito através de exames de imagem, como a RNM e a $\mathrm{TC}$, que revelam a causa da constrição do nervo facial, com exceção dos casos de origem idiopática. $\mathrm{O}$ trata-
Tabela 1: Diagnóstico Diferencial dos Tremores Involuntários

\begin{tabular}{|c|c|}
\hline $\mathrm{EH}$ & $\begin{array}{l}\text { Contrações clônicas intermitentes } \\
\text { dos músculos invervados pelo ner- } \\
\text { vo facial, é usualmente unilateral } \\
\text { e os fatores agravantes incluem o } \\
\text { estresse e a fadiga; os movimentos } \\
\text { persistem durante o sono. }\end{array}$ \\
\hline $\begin{array}{l}\text { Espasmo facial } \\
\text { psicogênico }\end{array}$ & $\begin{array}{l}\text { Contrações intermitentes ou cons- } \\
\text { tantes, os movimentos são não pa- } \\
\text { dronizados e envolvem a face e toda } \\
\text { a parte do corpo do lado acometido. } \\
\text { Aumenta com o estresse, ansieda- } \\
\text { de, fadiga e alivia com tratamento } \\
\text { placebo. }\end{array}$ \\
\hline Tiques & $\begin{array}{l}\text { Movimentos rápidos e estereotipa- } \\
\text { dos que se assemelham a movimen- } \\
\text { tos normais e coordenados. Podem } \\
\text { envolver músculos não invervados } \\
\text { pelo nervo facial. Geralmente en- } \\
\text { volvem a face e membros e são } \\
\text { capazes de serem reproduzidos e } \\
\text { suprimidos voluntariamente. }\end{array}$ \\
\hline Blefarospasmo & $\begin{array}{l}\text { Movimento distônico do músculo } \\
\text { orbicular do olho, usualmente, é } \\
\text { bilateral e aumenta com o estresse, } \\
\text { ansiedade e fadiga e melhora du- } \\
\text { rante o sono. }\end{array}$ \\
\hline Mioquimia & $\begin{array}{l}\text { Movimentos ondulantes dos mús- } \\
\text { culos faciais, principalmente no } \\
\text { orbicular. }\end{array}$ \\
\hline
\end{tabular}

Contratura fa- Paresia e contração contínua no cial, parética e lado envolvido, e não com relaxaespástica. mento entre as contrações, como ocorre no EH. Geralmente está relacionada com doenças do tronco cerebral.

Síndrome de Uma associação do blefarospasmo Meige com movimentos discinéticos da região inferior da face.

mento é dependente da etiologia da doença e consiste no procedimento cirúrgico de descompressão do nervo facial - para resolução do EH secundário - ou na administração de fármacos benzodiazepínicos com o intuito de amenizar as contrações exacerbadas. Há ainda a possibilidade da aplicação de toxina botulínica do tipo A com intervalo de 1 a 4 meses, visando oferecer um melhor bem-estar e conforto ao paciente com dada patologia. Vale destacar, que o tema ainda é pouco estudado e relatado, sendo importante a realização de novos 
estudos e pesquisas, a fim de descrever a patogênese da doença e opções terapêuticas, principalmente, para que o EH possa ser mais conhecido, melhor abordado e identificado.

\section{Referências}

1- Barbosa ER, Costa MDL, Staut CC, Bacheschi L, Bittar MS. Espasmo hemifacial familiar: relato de dois casos. Arq. Neuro-Psiquiatr. $1998 ; 56(1): 111-115$

2- $\quad$ Batisti JP, Kleinfelder AD, Galli NB, Moro A, Munhoz RP, Teive HA. Treatment of hemifacial spasm with botulinum toxin type a: effective, long lasting and well tolerated. Arq. Neuro-Psiquiatr. 2017; 75(2):87-91.

3- Lefaucheur JP, Daamer NB, Sangla S, Guerinele CL. Diagnosis of primary hemifacial spasm. Neurochirurgie. In Press 2018.

4- Borges V, Ferraz BH. Espasmo hemifacial. Rev. Neurociências. 1998; 9(1): 5-8.

5- $\quad$ Tan NC, Chan LL, Tan EK. Hemifacial spasm and involuntary facial movements. Q J Med. 2002;95(8):493-500.

6- Lasalvia CGG, Pereira LS, Cunha MC, Kitadai SPS. Custos e eficácia da toxina botulínica tipo A no tratamento do blefaroespasmo essencial e espasmo hemifacial. Arq. Bras. Oftalmol. 2006;69(5):701-705.

7- Machado FCN, Fregni F, Campos CR, Limongi JCP. Espasmo hemifacial bilateral: relato de caso. Arq. Neuro-Psiquiatr. 2003; 61(1):115118.

8- BentoRF, BogarP, Caldas SS, MaroneSAM, Miniti A. Espasmo Hemifacial: dez anos de experiência. Revista Brasileira de Otorrinolaringologia. 1991;57(3):105-9.

9- ArnezJCL, Arostegui PB, Fernández AMCA. Uso De Toxina Botulínica En Espasmo Hemifacial: A Propósito De Un Casoabout A Case. Rev. Méd. La Paz. 2016;22(2):56-59.

10- Lefaucheur JP. New insights into the pathophysiology of primary hemifacial spasm. Neurochirurgie. In Press 2018.

11- Safarpour Y, Jabbari B. Botulinum Toxin Treatment of Movement Disorders. Curr Treat Options. Neurol. 2018; 20(4):3-17.

12- Ferreira M, Walcott BP, Nahed BV, Sekhar LN. Vertebral artery pexy for microvascular decompression of the facial nerve in the treatment of hemifacial spasm. Journal of Neurosurgery. 2011;114(6):1800-1804.

13- Sindou M, Mercier P. Microvascular decompression for hemifacial spasm: Outcome on spasm and complications. A review. Neurochirurgie. In Press 2018

14- Camargos S, Costa M, Filogônio I, Cardoso F. Manual para o Diagnóstico dos Distúrbios de Movimento. São Paulo: Editora e Eventos Omnifarma Ltda; 2012.

15- Yaltho TC, Jankovic J. The many faces of hemifacial spasm: Differential diagnosis of unilateral facial spasms. Movement Disorders. 2011; 26(9): 1582-1592. 